\title{
Distribution Analysis of Rated Power for 6m Passenger Car
}

\author{
Guoliang Dong ${ }^{1, \mathrm{a}^{*}}$, Shuquan $\mathrm{Xu}^{1, \mathrm{~b}}$ \\ ${ }^{1}$ Research Institute of Highway Ministry of Transport, Beijing, China \\ agl.dong@rioh.cn, bsq.xu@rioh.cn
}

\begin{abstract}
Keywords: passenger car, rated power, mismatch, hypothesis distribution, EDF, goodness test Abstract. Thousands of motor models are assembled in the vehicles. It is necessary to study the distribution characteristics of motor rated power to check the vehicle plants have obeyed the optimal designed methods. Some passenger car samples of body length shorter than $6 \mathrm{~m}$ have been analyzed. Construct statistics for different hypotheses distributions, estimate their values, test the goodness of these distribution hypotheses and determine their distribution characteristics.
\end{abstract}

\section{Introduction}

According to some statistics, there are many thousands of passenger car models in the market. Improper configuration design will result in the power mismatch, weak safety, performance loss and service life decrease [1] etc. Currently, rated power mismatch is a common and serious problem. In order to study present situation of power mismatch, we should acquire as many as possible passenger car models and sort out them by the body length to study the distribution character of the motor rated power. Car models of body length shorter than $6 \mathrm{~m}(\mathrm{~L}<=6 \mathrm{~m})$ have been analyzed. Assuming that motor rated power follows some distributions, construct statistics of various distributions, estimate values and test the goodness of these distribution hypotheses [2].

\section{Analysis method}

\section{Category}

The proper motor rated power is relative to the car body length. With constant sample size, a narrower length range will provide high precision of the typical value but reduce the sample quantity of each category. By reference to the category method of some standards [3], the vehicle models can be classified as almost $1 \mathrm{~m}$ interval for each category which results in a proper precision with a necessary sample size for each category.

\section{Samples}

Vehicle parameters can be acquired from many ways such as books, vehicle plants, automotive websites [4], vehicle forums, auto $4 \mathrm{~S}$ shops etc. The most authoritative data source is vehicle plant. Hundreds of $\mathrm{L}<=6 \mathrm{~m}$ passenger car models have been acquired from vehicle plants. In order to avoid interference of one model corresponding to various configurations (one model assembled with the same motor types), the same model with various configurations should be merged into one line.

\section{Data analysis}

\section{Distribution character}

Studying the scatter diagram and frequency chart of the rated power, the distribution of data focusing in the middle and decreasing on both sides is significant. According to the statistics theory, normal distribution, lognormal distribution, Weibull distribution and Gamma distribution all comply with the above feature [5,6]. 


\section{Hypothesis distribution theory}

There are many methods to construct statistic. The statistic based on the difference between the empirical distribution function (EDF) and the theoretical distribution function is called EDF statistic [7]. For continuous vary, the test of goodness of fit is better than $\chi^{2}$ test. And EDF statistics have nothing to do with the group methods [8]. Follow the three steps to study the distribution of motor power. (1) Compute the estimated parameter values of hypothesis function using maximum likelihood method (MLE). (2) Construct $A^{2}$-Statistics, $W^{2}$-Statistics and $D$-Statistics. (3) Test the goodness of fit check of the hypothesis distribution.

D-Statistics [9]:

$$
D_{n}=\sup _{\forall x \in R}\left[F_{n}(x)-F(x, \theta)\right]
$$

Where $\forall \mathrm{x}$ denotes with any $\mathrm{x}, \mathrm{R}$ denotes the range over of $\mathrm{x}, \mathrm{F}_{\mathrm{n}}\left(\mathrm{x}_{\mathrm{s}} \theta\right)$ denotes the theoretical distribution function of $\mathrm{X}, \mathrm{F}_{\mathbf{n}}(\theta)$ denotes the empirical distribution function of $\mathrm{X}$.

$A^{2}$-Statistics, $W^{2}$-Statistics can be calculated by Eq. 1 and Eq.2 $[10,11]$.

$$
A^{2}=n \int_{-\infty}^{+\infty}\left[F_{n}(x)-F(x, \theta)\right]^{2}\{F(x, \theta)[1-F(x, \theta)]\}^{-1} d F(x, \theta)
$$

$$
W^{2}=n \int_{-\infty}^{+\infty}\left[F_{n}(x)-F(x, \theta)\right]^{2} \mathrm{dF}(\mathrm{x}, \theta)
$$

Set $\mathrm{Z}=\mathrm{F}(\mathrm{X}, \theta), \quad A^{2}$-Statistics, $W^{2}$-Statistics and, $D$-Statistics can be computed by Eq.4-Eq. 6 .

$$
\begin{aligned}
& \left\{\begin{array}{l}
{D_{n}{ }^{+}}^{+}=\max _{\forall i \in(1,2 \mathrm{~L} n)}\left[\frac{i}{n}-z_{(i)}\right] \\
D_{n}{ }^{-}=\max _{\forall i \in(1,2 \mathrm{~L} n)}\left[z_{(i)}-\frac{i-1}{n}\right] \\
D_{n}=\max \left[D_{n}{ }^{+}, D_{n}{ }^{-}\right]
\end{array}\right. \\
& W_{n}^{2}=\sum_{i=1}^{n}\left[z_{[i]}-\frac{21-1}{2 n}\right]^{2}+\frac{1}{12 n}
\end{aligned}
$$




$$
A_{n}^{2}=-\frac{1}{n}\left\{\sum_{i=1}^{\mathrm{n}}(2 \mathrm{n}-1)\left[\ln \left[\mathbf{z}_{(\mathrm{j})}-\ln \left(1-\mathrm{z}_{(\mathrm{n}+1-\mathrm{i})}\right)\right]\right\}-\mathrm{n}\right.
$$

Considering that there are unknown variables in parameters, EDF Statistics can be adjusted by the sample size as Table 1 .

Table 1. The adjusted EDF statistics by sample size

\begin{tabular}{|l|l|l|l|}
\hline \multirow{2}{*}{} & \multicolumn{2}{|c|}{$T_{n}^{\text {m, }}$, the correction of statistic $T_{n}$ by sample size } & \multirow{2}{*}{$D^{*}$} \\
\cline { 2 - 4 } & $A^{*}$ & $W^{*}$ & \\
\hline Normal & $A_{n}^{2}\left(1+\frac{0.75}{n}+\frac{2.25}{n^{2}}\right)$ & $W_{n}^{2}\left(1+\frac{0.5}{n}\right)$ & $D_{n}\left(\sqrt{n}-0.01+\frac{0.85}{\sqrt{n}}\right)$ \\
\hline Wognormal & $A_{n}^{2}\left(1+\frac{0.2}{\sqrt{n}}\right)$ & $W_{n}^{2}\left(1+\frac{0.2}{\sqrt{n}}\right)$ & \\
\hline Gamma & $A_{n}^{2}+\frac{1}{n}\left(0.2+\frac{0.3}{n}\right)$ & $\frac{1.8 n V_{n}^{2}-0.14}{1.8 n-1}$ & \\
\hline
\end{tabular}

Compare the statistic value with the adjusted value to judge if the EDF complying with the distribution hypotheses or not.

\section{Results and discussion}

\section{Data processing}

MATLAB is an efficient tool for scientific computation. By editing some lines of program code, we can compute parameter estimation of hypothesis distribution and EDF statistic. EDF statistic and the correction of rated power for $\mathrm{L}<=6 \mathrm{~m}$ passenger car are shown in Table 2 (Sample size $=584)$.

Table 2 EDF statistics and the correction of rated power for $\mathrm{L}<=6 \mathrm{~m}$ passenger car

\begin{tabular}{|c|c|c|c|c|c|c|}
\hline $\begin{array}{c}\text { statistic } \\
\text { hypothesis }\end{array}$ & $A^{2}$ & $W^{2}$ & $D$ & $A^{*}$ & $W^{*}$ & $D^{*}$ \\
\hline Normal & 6.5521 & 1.2973 & 0.1314 & 6.5605 & 1.2984 & 3.1792 \\
\hline Lognormal & 7.5978 & 1.5278 & 0.1260 & 7.6077 & 1.5291 & 3.0469 \\
\hline Weibull & 10.1764 & 1.9275 & 0.1613 & 10.1895 & 1.9292 & 3.9021 \\
\hline Gamma & 6.9541 & 1.3956 & 0.1171 & 6.9630 & 1.3968 & 2.8322 \\
\hline
\end{tabular}




\section{Test results}

The critical value at the significance level for the test statistics are shown in Table 3.

Table 3 . The critical value at the significance level for the test statistics

\begin{tabular}{|c|c|c|c|}
\hline significance level $\alpha$ & $A^{2}$ & $W^{2}$ & $\sqrt{n D}$ \\
\hline 0.25 & - & 0.472 & 0.074 \\
\hline 0.10 & 0.819 & 0.632 & 0.102 \\
\hline 0.05 & 0.895 & 0.754 & 0.126 \\
\hline 0.025 & 0.995 & 0.875 & 0.148 \\
\hline 0.01 & 1.035 & 1.036 & 0.178 \\
\hline
\end{tabular}

Comparing the adjusted $A^{2}, W^{2}, D$ with the critical value for different significance level (If

the statistics value is less than the critical value, the hypothesis distribution is accepted), we conclude that the distribution of rated power of $\mathrm{L}<=6 \mathrm{~m}$ passenger car smaples complies with no hypotheses distribution.

\section{Conclusion}

We have studied the distribution character of some passenger car model samples and computed the EDF for each hypothesis distribution. But the results are not good.

By studying the distribution characteristics of $\mathrm{L}<=6 \mathrm{~m}$ passenger car sample models, we can draw some conclusions:.

a) The motor power doesn't conform to the assumed hypotheses distribution such as normal distribution, lognormal distribution etc.

b) It is possible that the motor plants haven't obeyed the optimal design rules

\section{References}

[1] Zhisheng Yu, Theory of Automobile, China Machine Press,2009.

[2] Cong Dong, Haiwu Rong, Renwei Xia, The Distribution Model of Fatigue Life and its Good-of-Fit Test, Chinese J. of Aeronautics (1995), 8(3), pp.185-190.

[3] JT 711-2008 Limits and Measurement Methods of Fuel Consumption for Commercial Vehicle for Passenger Transportation.

[4] Information on http://www.xcar.com.cn/

[5] Zou Sheng, Shiqian Xie, Chengyi Pan,Probability and Mathematical Statistics, Higher Education Press ,2010.

[6] Junping Jia, Statistics, China Renmin University Press Co., LTD ,2015.

[7] Zhenhai Yang, Weihu Cheng, Junjian Zhang, Goodness of Fit Test, Science Press,2001.

[8] Cong Dong, Modern structural system reliability theory and its application, Science Press,2001.

[9] Peter J. Bickel, Kjell A.Doksum, Mathematical Statistics Basic Ideas and Selected Topics, Prentice-Hall ,Inc., 2001.

[10] Wei Zhang,Shenglin Yu,Gong Zhang, Anderson-Darling test for clutter distribution identification, Chinese Journal of Scientific Instrument, Vol.30,2009,pp.631-635 
[11] Paul A. Tobias \& David C. Trindade, Applied Reliability, third ed., CRC Press, Taylor \& Francis Group,2012. 